\title{
Estrutura diamétrica da vegetação arbustivo-arbórea no entorno de nascentes da bacia hidrográfica do rio Piauitinga, Salgado - SE, Brasil
}

\author{
Diogo Gallo de Oliveira * \\ Robério Anastácio Ferreira \\ Anabel Aparecida de Mello \\ Ednei Santos de Almeida \\ Universidade Federal de Sergipe \\ Avenida Marechal Rondon s/n, São Cristóvão, CEP 49100-000, Sergipe - SE, Brasil \\ * Autor para correspondência \\ diogo_gallo@hotmail.com
}

Submetido em 31/08/2012

Aceito para publicação em 21/03/2013

\section{Resumo}

Este estudo foi realizado com o propósito de analisar a estrutura diamétrica da vegetação ciliar em nascentes da bacia hidrográfica do rio Piauitinga, localizadas no município de Salgado-SE. Foram analisadas 14 nascentes, mediante classificação quanto ao estado de conservação e ao tipo de reservatório, sendo divididas em 4 categorias: 1 preservada pontual (PrP), 1 perturbada pontual (PP), 6 degradadas pontuais (DP) e 6 degradadas difusas (DD). O levantamento foi realizado por meio de censo populacional da vegetação em um raio de $50 \mathrm{~m}$ no entorno das nascentes, equivalente a 0,79 ha, onde foram registrados todos os indivíduos arbustivo-arbóreos vivos com diâmetro à altura do peito $(\mathrm{DAP}) \geq 5,0 \mathrm{~cm}$. Os indivíduos foram distribuídos em classes diamétricas, com intervalos de $2,5 \mathrm{~cm}$. A nascente preservada pontual e a perturbada pontual apresentaram estrutura diamétrica característica de florestas inequiâneas, com a forma de "J invertido". As nascentes degradadas difusas e pontuais não apresentaram o padrão de "J invertido", com exceção da DD2, talvez reflexo do maior número de indivíduos nessa nascente. As intensas perturbações geradas na área das nascentes, que atualmente se encontram degradadas, promoveram o empobrecimento da regeneração natural e, consequentemente, prejudicou o recrutamento de indivíduos nas classes iniciais de diâmetro.

Palavras-chave: Conservação de nascentes; Fitossociologia; Vegetação ciliar

\section{Abstract}

Diametric structure of the shrub and tree vegetation around the headwaters in the Piauitinga River drainage basin, Salgado - Sergipe, Brazil. This study was carried out in order to analyze the diametric structure of the ciliary vegetation in headwaters in the Piauitinga River drainage basin, located at the town of Salgado, Sergipe, Brazil. One analyzed 14 headwaters, using a classification with regard to the conservation status and the type of reservoir, being divided into 4 categories: 1 punctual preserved (PPr), 1 punctual disturbed (PD), 6 punctual degraded (PD), and 6 diffuse degraded (DD). The survey was conducted through a population census of vegetation in a $50 \mathrm{~m}$ radius around the headwaters, equivalent to 0.79 ha, where one recorded all living shrub and tree individuals with diameter at breast height $(\mathrm{DBH}) \geq 5.0 \mathrm{~cm}$. The individuals were distributed into 
diametric classes, at $2.5 \mathrm{~cm}$ intervals. The punctual preserved and the punctual disturbed headwaters showed a diametric structure characteristic of heterogeneous forests, with an "inverted J" shape. The diffuse and punctual degraded headwaters didn't show the "inverted J" pattern, except for DD2, perhaps reflecting the higher number of individuals in this headwater. The intense disturbance generated in the headwater areas, which are currently degraded, promoted the impoverishment of natural regeneration and, as a result, compromised the recruitment of individuals in the early diameter grades.

Key words: Ciliary vegetation; Phytosociology; Preservation of headwaters

\section{Introdução}

Vegetação ciliar, de modo particular, é conceituada por Ab'Saber (2004), como toda aquela associada às margens de nascentes ou cursos d'água, independente de sua extensão, localização e composição florística. Este tipo de vegetação apresenta características fisionômicas distintas, definidas por uma interação complexa de fatores dependentes das condições ambientais ciliares (edafoclimáticas e biológicas) (RODRIGUES, 2004).

A existência da flora em áreas ciliares é fundamental para a proteção dos recursos hídricos, equilíbrio climático local e como reserva da biodiversidade (MARTINS, 2007). Desta forma, a proteção da vegetação presente nas áreas ciliares, no Brasil, está prevista em legislação desde 1965 com a criação do Código Florestal (Código Florestal - Lei no 4.771/65), permitindo assim, a preservação da biodiversidade presente nestes ambientes (BRASIL, 2010).

A bacia hidrográfica do rio Piauitinga, localizada na região citrícola do centro-sul do estado de Sergipe representa notável importância para os municípios que nela estão inseridos, principalmente pela qualidade da água e capacidade de abastecimento (MOREIRA, 2008). Entretanto, encontra-se atualmente com grande parte dos seus mananciais em avançado estado de degradação, ocasionado principalmente pela produção agrícola irracional, sem o uso de práticas conservacionistas e a criação de gado sem manejo adequado nas áreas ciliares (SANTOS, 2009).

Desta forma, torna-se essencial o conhecimento da dinâmica vegetacional que ocorre nesses ambientes, os quais são marcados pela heterogeneidade florística originada por condições edafoclimáticas, hidrológicas e ecológicas específicas (RODRIGUES; NAVE, 2004). Tal conhecimento pode ser obtido por meio de estudos fitossociológicos, que se referem à estrutura horizontal e vertical da vegetação e que geram subsídios para elaboração de planos de restauração e ou recuperação de ambientes degradados (MARANGON et al., 2007; FERREIRA et al., 2011; OLIVEIRA et al., 2012).

De acordo com Machado et al. (2010), grande parte da estrutura de uma floresta pode ser explicada por meio da avaliação de sua distribuição diamétrica, a qual é definida pela caracterização do número de indivíduos por unidade de área e por intervalo de classe de diâmetro. O estudo da estrutura arbórea em comunidades propicia informações a respeito do seu desenvolvimento, grau de perturbação, níveis de estágio de desenvolvimento e sucessão da comunidade (ALVES- JÚNIOR et al., 2007).

Entretanto, Virillo et al. (2011), após analisarem dados sobre a estrutura de cinco espécies lenhosas de Cerrado, por um período de doze anos afirmaram que medidas de estrutura de tamanho não devem ser usadas como base para decisões de manejo sem que análises demográficas mais apropriadas sejam realizadas.

Segundo Imaña-Encinas et al. (2009), os estudos sobre a estrutura diamétrica da comunidade florestal e de suas espécies por separado podem fornecer subsídios para um diagnóstico de comportamento de regeneração, mortalidade e de alguns eventos internos e externos ao ambiente florestal.

Por meio da avaliação da estrutura em comunidades vegetais, pode-se identificar o comportamento ecológico e o padrão de cada população, possibilitando a compreensão das características de cada espécie e o maior entendimento das estratégias de regeneração natural, crescimento e sobrevivência (ALVES-JÚNIOR et al., 2007).

Assim, este estudo foi realizado com o objetivo de analisar a estrutura diamétrica do estrato arbustivo- 
arbóreo e a semelhança florística da vegetação ciliar em nascentes da bacia hidrográfica do rio Piauitinga, localizadas no município de Salgado-SE.

\section{Material e Métodos}

O estudo foi conduzido na região centro-sul do Estado de Sergipe, no município de Salgado, na área da bacia hidrográfica do rio Piauitinga, que integra a bacia hidrográfica do rio Piauí (Figura 1). O município de Salgado localiza-se geograficamente nas coordenadas $11^{\circ} 01^{\prime} 50^{\prime \prime}$ e $11^{\circ} 09^{\prime} 57^{\prime} \mathrm{S}$; e $37^{\circ} 28^{\prime} 05^{\prime \prime}$ e $37^{\circ} 32^{\prime} 51^{\prime \prime} \mathrm{W}$, em uma altitude de $102 \mathrm{~m}$, abrangendo uma área correspondente a $255,8 \mathrm{~km}^{2}$ com contribuição de $56,05 \%$ do seu território à área da bacia hidrográfica. De acordo com a classificação climática de Thornthwaite e Mather, apresenta dois tipos climáticos: C1A'a' - Megatérmico Subúmido e C2A'a' - Megatérmico Subúmido Úmido (FONTES et al., 1999), precipitação média anual de 1.249 mm e temperatura média anual de $24,6^{\circ} \mathrm{C}$ com período chuvoso de março a julho (BOMFIM et al., 2002).

O relevo apresenta predominância de feições planas de pequena altitude, condicionadas pela disposição dos sedimentos terciários do Grupo Barreiras, os quais recobrem grande parte da superfície do município, além de tabuleiro costeiro com superfície tabular erosiva e relevos dissecados em colinas, cristas e interflúvios tabulares (BOMFIM et al., 2002).

Os solos da região são classificados como Argissolo Vermelho Amarelo, Latossolo Vermelho Amarelo Distrófico e Neossolo Flúvico (SERGIPE, 2010), de acordo com o Sistema Brasileiro de Classificação do Solo (EMBRAPA, 2006).

A vegetação presente na região, segundo o sistema de classificação de Veloso et al. (1991), pode ser qualificada como Floresta Estacional Semidecidual Submontana e transição entre Floresta Estacional e áreas que sofreram antropização com a agropecuária (MMA, 2006).

A localização das nascentes foi obtida com auxílio da carta do Atlas Digital de Sergipe (SERGIPE, 2010), na qual foi considerada como nascente cada início de tributário da bacia hidrográfica, com um aparelho GPS (Global Position System), modelo Garmim Etrex Vista, e com o auxílio de um morador da região, pelo fato de conhecer a localização das nascentes.

FIGURA 1: Mapa de localização do município de Salgado, na bacia hidrográfica do rio Piauitinga, Sergipe.

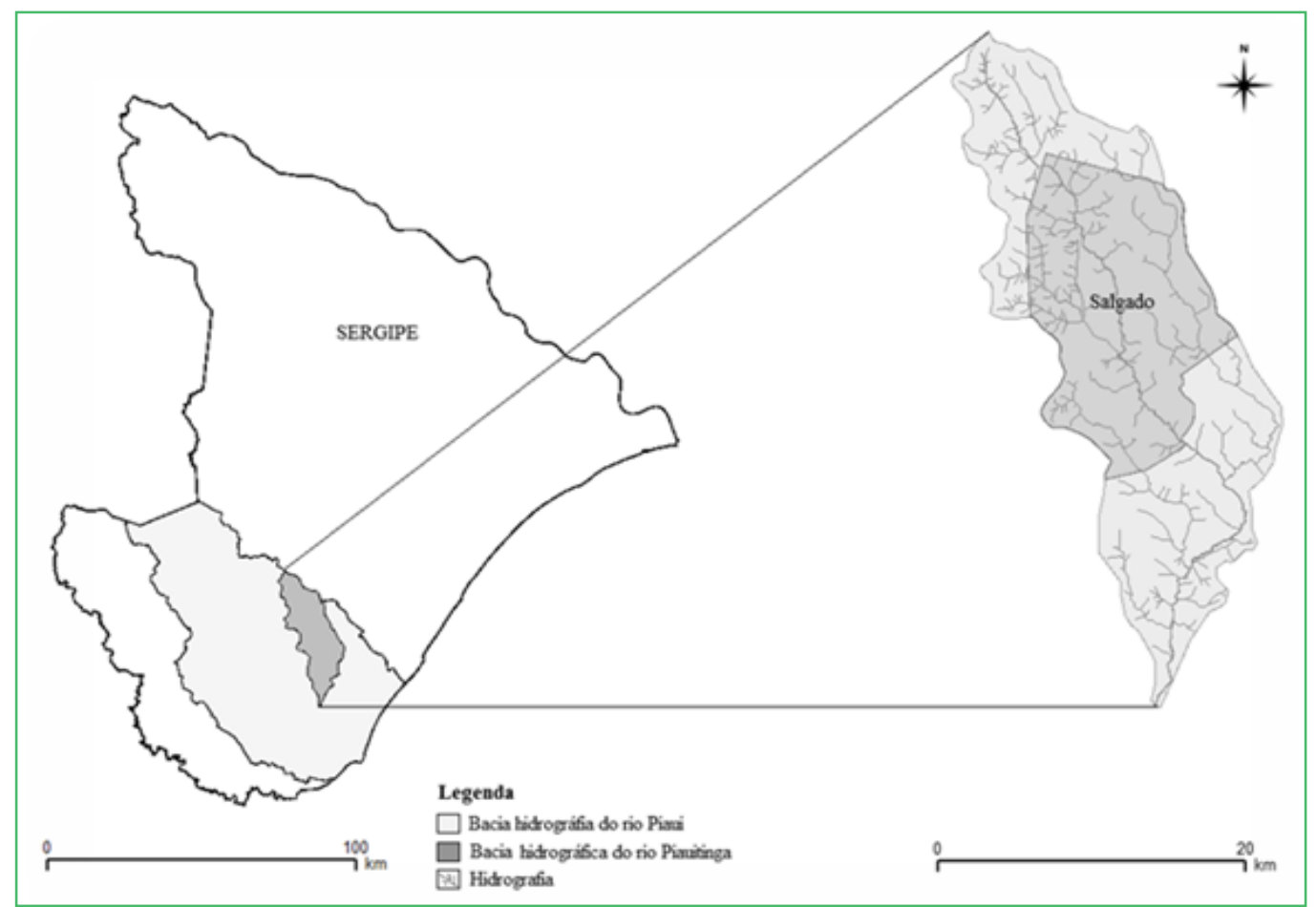


Foram analisadas 14 nascentes, mediante classificação das mesmas quanto ao tipo de reservatório a que estavam associadas, em pontuais e difusas (CASTRO, 2007) e, quanto ao estado de conservação em preservadas, perturbadas e degradadas (Tabela 1), conforme metodologia utilizada por Pinto et al. (2005), formando quatro categorias de estudo: uma preservada pontual (PrP), uma perturbada pontual (PP), seis degradadas pontuais (DP) e seis degradadas difusas (DD). As nascentes receberam um número código, de acordo com a ordem de avaliação em campo, onde a letra inicial representa o estado de conservação, a segunda o tipo de reservatório associado à nascente, e o número representa a ordem de avaliação para aquela categoria.

O levantamento florístico e estrutural do estrato arbustivo-arbóreo foi realizado por meio de censo populacional da vegetação no entorno das nascentes, em área equivalente a 0,79 ha estabelecida dentro de um raio de $50 \mathrm{~m}$ correspondente à faixa mínima prescrita pelo Código Florestal - Lei no 4.771/65. Em cada nascente foram identificados e registrados todos os indivíduos arbustivo-arbóreos vivos com diâmetro à altura do peito (DAP a 1,30 $\mathrm{m}$ do nível do solo) $\geq 5,0 \mathrm{~cm}$, incluindose também os bifurcados, caso atendessem ao nível de inclusão mínimo estabelecido. Foram medidos o DAP e a altura total de cada indivíduo, com auxílio de fita diamétrica e vara telescópica $(10 \mathrm{~m})$, respectivamente. Os indivíduos foram etiquetados com plaquetas de alumínio numeradas, e anotados o nome regional da espécie, o diâmetro e a altura total.

O material botânico coletado foi devidamente herborizado e identificado por comparação em literatura taxonômica especializada e com auxílio de especialistas do Herbário do Instituto do Meio Ambiente do Estado de Alagoas (MAC) e do Herbário da Universidade Federal de Sergipe (ASE). Todo material botânico foi depositado no herbário ASE. As espécies foram classificadas de acordo com o Sistema Angiosperm Phylogeny Group III (APGIII, 2009) e a confirmação da grafia e da autoria foram obtidas a partir de consulta ao banco de dados do Missuori Botanical Garden's VAST - MOBOT (www. mobot.mobot.org/W3T/search/vast.html) e também na lista de espécies da flora do Brasil 2012 em: http:// floradobrasil.jbrj.gov.br/2012.

Para análise da estrutura horizontal do estrato arbustivo-arbóreo foram considerados os seguintes parâmetros fitossociológicos: densidade absoluta e relativa $\left(\mathrm{DA}_{\mathrm{i}}\right.$ e $\left.\mathrm{DR}_{\mathrm{i}}\right)$, dominância absoluta e relativa $\left(\right.$ DoA $_{i}$ e DoR $\left.R_{i}\right)$, frequência absoluta e relativa $\left(F_{i}\right.$ e FR $\left.{ }_{i}\right)$ e o índice de valor de importância (IVI) (MUELLERDOMBOIS; ELLENBERG, 1974).

TABELA 1: Caracterização das nascentes avaliadas na bacia hidrográfica do rio Piauitinga, Salgado, SE, quanto ao estado de conservação, tipo de reservatório, coordenadas geográficas (latitude e longitude) e altitude.

\begin{tabular}{llllll}
\hline Código & $\begin{array}{l}\text { Estado de } \\
\text { conservação }\end{array}$ & Tipo de reservatório & Latitude & Longitude & Altitude (m) \\
\hline PrP1 & Preservada & Pontual & 662197 & 8780034 & 126 \\
PP1 & Perturbada & Pontual & 662983 & 8776784 & 113 \\
DD1 & Degradada & Difusa & 661912 & 8779524 & 105 \\
DD2 & Degradada & Difusa & 662664 & 8779404 & 115 \\
DD3 & Degradada & Difusa & 660989 & 8779190 & 110 \\
DD4 & Degradada & Difusa & 666720 & 8779686 & 99 \\
DD5 & Degradada & Difusa & 666516 & 8783682 & 119 \\
DD6 & Degradada & Difusa & 663218 & 8780302 & 125 \\
DP1 & Degradada & Pontual & 660582 & 8778680 & 91 \\
DP2 & Degradada & Pontual & 663360 & 8776564 & 106 \\
DP3 & Degradada & Pontual & 663543 & 8778942 & 103 \\
DP4 & Degradada & Pontual & 667711 & 8785180 & 133 \\
DP5 & Degradada & Pontual & 664914 & 8779090 & 113 \\
DP6 & Degradada & Pontual & 661942 & 8777158 & 110 \\
\hline
\end{tabular}


A análise da estrutura diamétrica foi realizada com auxílio do software Mata Nativa 2.10® (CIENTEC, 2006). Os indivíduos foram distribuídos em classes diamétricas, sendo que o centro da primeira classe diamétrica foi de $6,3 \mathrm{~cm}$, com intervalos de $2,5 \mathrm{~cm}$.

\section{Resultados}

No levantamento estrutural do estrato arbóreoarbustivo das 14 nascentes foram registrados 1874 indivíduos, distribuídos em 101 espécies (12 não identificadas), 75 gêneros e 41 famílias botânicas (Tabela 2), resultando em uma densidade média de 170 indivíduos/ha e a área basal de 2,64 $\mathrm{m}^{2} /$ ha. Das 12 espécies não identificadas, sete foram indicadas apenas pela família.

As famílias botânicas mais expressivas, em todas as nascentes, em termos de indivíduos foram: Malpighiaceae (16,14\%), Anacardiaceae (12,84\%), Myrtaceae (10,96\%), Lecythidaceae (9,93\%), Urticaceae (7,66\%), Mimosaceae (7,61\%) e Fabaceae (6,31\%), somando $71,45 \%$ do total de indivíduos amostrados. Em relação ao número de espécies, as famílias mais ricas foram: Myrtaceae (10), Mimosaceae (8), Fabaceae
(6), Sapindaceae (5), Anacardiaceae (4), Malvaceae (4), Rubiaceae (4), Annonaceae (3) e Caesalpinaceae (3).

Quanto ao índice de valor de importância (IVI), as dez espécies com maior destaque, em ordem decrescente, foram: Byrsonima sericea, Tapirira guianensis, Cecropia pachystachya, Eschweilera ovata, Bowdichia virgilioides, Inga laurina, Anacardium occidentale, Eugenia sp., Cupania zanthoxyloides e Protium heptaphyllum, representando 51,32\% do total de indivíduos.

Foram registradas cinco espécies exóticas na área das nascentes degradadas: Mangifera indica L., Arthocarpus integrifolia Cham., Syzygium jambolanum (Lam.) DC., Terminalia catappa L. e Leucaena leucocephala (Lam.) de Wit.

A nascente preservada pontual $(\mathrm{PrP})$ e a perturbada pontual (PP) apresentaram estrutura diamétrica característica de florestas inequiâneas, com a forma de um "J invertido", a qual mostra uma maior concentração de indivíduos de menor diâmetro nas classes iniciais e diminuição acentuada no sentido das maiores classes (Figura 2). Os centros das classes de diâmetros compreendidas entre 6,3 e $8,8 \mathrm{~cm}$, juntos compreenderam respectivamente, $74 \%$ e $70 \%$ do total dos indivíduos.

TABELA 2: Estimativa dos parâmetros fitossociológicos de 14 nascentes estudadas na bacia hidrográfica do Rio Piauitinga, município de Salgado, SE. DA: densidade absoluta (indivíduos/ha); FA: frequência absoluta (\%); DoA: dominância absoluta ( $\left.\mathrm{m}^{2} / \mathrm{ha}\right)$; IVI: índice de valor de importância.

\begin{tabular}{llcccc}
\hline Famílias & Espécies & DA & FA & DoA & IVI \\
\hline Malpighiaceae & Byrsonima sericea DC. & 27,19 & 85,71 & 0,267 & 30,08 \\
Anacardiaceae & Tapirira guianensis Aublet & 16,92 & 92,86 & 0,399 & 29,39 \\
Urticaceae & Cecropia pachystachya Trécul & 12,91 & 92,86 & 0,359 & 25,50 \\
Lecythidaceae & Eschweilera ovata (Cambess.) Miers & 15,37 & 78,57 & 0,130 & 17,60 \\
Fabaceae-Faboideae & Bowdichia virgilioides Kunth & 6,73 & 64,29 & 0,131 & 11,90 \\
Fabaceae-Mimosoideae & Inga laurina Willd & 4,55 & 50,00 & 0,113 & 9,29 \\
Anacardiaceae & Anacardium occidentale L. & 2,46 & 35,71 & 0,144 & 8,57 \\
Myrtaceae & Eugenia sp. & 8,55 & 42,86 & 0,036 & 8,37 \\
Sapindaceae & Cupania zanthoxyloides Cambess. & 5,73 & 57,14 & 0,018 & 6,69 \\
Burseraceae & Protium heptaphyllum (Aubl.) Marchand. & 4,55 & 57,14 & 0,031 & 6,51 \\
Anacardiaceae & Mangifera indica L. & 1,09 & 28,57 & 0,116 & 6,36 \\
Fabaceae-Caesalpinioideae & Cassia grandis L.f. & 1,00 & 7,14 & 0,143 & 6,34 \\
Fabaceae-Mimosoideae & Stryphnodendron pulcherrimum (Willd.) Hochr. & 1,64 & 35,71 & 0,096 & 6,27 \\
Apocynaceae & Himatanthus obovatus (Müll. Arg.) Woodson & 4,64 & 42,86 & 0,035 & 6,04 \\
\hline
\end{tabular}




\begin{tabular}{|c|c|c|c|c|c|}
\hline Fabaceae-Faboideae & Andira fraxinifolia Benth. & 1,18 & 42,86 & 0,085 & 5,90 \\
\hline Fabaceae-Mimosoideae & Inga vera Willd & 3,18 & 42,86 & 0,035 & 5,17 \\
\hline Myrtaceae & Calyptranthes cf. pauciflora O. Berg & 3,00 & 50,00 & 0,015 & 4,65 \\
\hline Annonaceae & Xylopia frutescens Aublet & 2,09 & 50,00 & 0,012 & 4,00 \\
\hline Myrtaceae & Campomanesia xanthocarpa O. Berg. & 2,37 & 42,86 & 0,007 & 3,66 \\
\hline Malvaceae & Guazuma ulmifolia Lam. & 1,27 & 42,86 & 0,021 & 3,52 \\
\hline Lauraceae & Ocotea glomerata (Nees) Mez & 2,46 & 28,57 & 0,018 & 3,45 \\
\hline Fabaceae-Mimosoideae & sp. 1 & 0,55 & 14,29 & 0,064 & 3,41 \\
\hline Moraceae & Arthocarpus integrifolia Cham. & 0,73 & 21,43 & 0,043 & 3,06 \\
\hline Asteraceae & Gochnatia oligocephala (Gardner) Cabrera & 2,09 & 21,43 & 0,012 & 2,68 \\
\hline Fabaceae-Mimosoideae & Abarema cochliacarpos (Campo) Barneby \& J. W. grimes & 1,09 & 28,57 & 0,019 & 2,68 \\
\hline Sapindaceae & Allophyllus cf. edulis (St. Hil.) Radlk. & 1,18 & 35,71 & 0,006 & 2,57 \\
\hline Rubiaceae & Genipa americana $\mathrm{L}$. & 1,09 & 21,43 & 0,023 & 2,50 \\
\hline Nyctaginaceae & Guapira tomentosa (Casar.) Lundell & 1,27 & 28,57 & 0,006 & 2,30 \\
\hline Lecythidaceae & Lecythis cf. pisonis Cambess. & 1,36 & 28,57 & 0,005 & 2,31 \\
\hline Boraginaceae & Cordia toqueve Aubl. & 1,82 & 14,29 & 0,011 & 2,17 \\
\hline Fabaceae-Faboideae & Lonchocarpus sericeus (Poir.) Kunth ex DC. & 1,73 & 7,14 & 0,02 & 2,09 \\
\hline Myrtaceae & Campomanesia dichotoma (O. Berg) Mattos & 0,82 & 28,57 & 0,006 & 2,02 \\
\hline Boraginaceae & Cordia nodosa Lam. & 0,73 & 28,57 & 0,003 & 1,88 \\
\hline Fabaceae-Mimosoideae & Piptadenia sp. & 0,82 & 14,29 & 0,019 & 1,85 \\
\hline Anacardiaceae & Thyrsodium spruceanum Benth. & 1,18 & 21,43 & 0,003 & 1,82 \\
\hline Sapindaceae & Cupania sp. & 0,91 & 21,43 & 0,007 & 1,80 \\
\hline Rubiaceae & Guettarda viburnoides Cham. \& Schltdl. & 1,09 & 21,43 & 0,005 & 1,81 \\
\hline Myrtaceae & Psidium guajava L. & 0,46 & 28,57 & 0,004 & 1,73 \\
\hline Primulaceae & Myrsine guianensis (Aubl.) Kuntze & 1,46 & 7,14 & 0,015 & 1,74 \\
\hline Rhamnaceae & Zizyphus joazeiro Mart. & 0,73 & 21,43 & 0,006 & 1,65 \\
\hline Nyctaginaceae & Guapira sp. & 0,36 & 28,57 & 0,003 & 1,65 \\
\hline Lamiaceae & Aegiphila verticillata Vell. & 0,82 & 21,43 & 0,004 & 1,62 \\
\hline Lamiaceae & Vitex rufescens A. Juss. & 0,27 & 21,43 & 0,012 & 1,62 \\
\hline Annonaceae & Duguetia gardneriana Mart. & 1,27 & 14,29 & 0,0015 & 1,59 \\
\hline Myrtaceae & Myrcia sp. & 1,18 & 14,29 & 0,005 & 1,56 \\
\hline Fabaceae-Mimosoideae & Mimosa tenuiflora (Willd.) Poir. & 0,82 & 14,29 & 0,008 & 1,46 \\
\hline Fabaceae-Faboideae & Machaerium hirtum (Vell.) Stellfeld & 0,55 & 21,43 & 0,004 & 1,46 \\
\hline Salicaceae & Casearia sylvestris $\mathrm{Sw}$. & 0,55 & 21,43 & 0,003 & 1,42 \\
\hline Chrysobalanaceae & sp.1 & 1,46 & 7,14 & 0,005 & 1,37 \\
\hline Rutaceae & Zanthoxylum rhoifolium Lam. & 0,36 & 21,43 & 0,002 & 1,29 \\
\hline Myrtaceae & Stenocalyx dysentericus (DC.) O. Berg & 0,36 & 21,43 & 0,002 & 1,28 \\
\hline Dilleniaceae & Curatella americana $\mathrm{L}$. & 0,64 & 14,29 & 0,006 & 1,26 \\
\hline Fabaceae-Caesalpinioideae & Bauhinia acuruana Benth. & 0,73 & 14,29 & 0,003 & 1,20 \\
\hline Schoepfiaceae & Schoepfia brasiliensis A.DC. & 0,64 & 14,29 & 0,003 & 1,14 \\
\hline Sapindaceae & Cupania cf. revoluta Radlk & 0,64 & 14,29 & 0,002 & 1,12 \\
\hline Indeterminada & Desconhecida 16 & 0,55 & 14,29 & 0,003 & 1,12 \\
\hline Sapotaceae & Manilkara rufula (Miq.) H.J. Lam & 0,46 & 14,29 & 0,003 & 1,04 \\
\hline Myrtaceae & Psidium guianense Pers. & 0,36 & 14,29 & 0,004 & 1,02 \\
\hline Melastomataceae & Tibouchina mutabilis Cong. & 0,36 & 14,29 & 0,003 & 0,98 \\
\hline Malvaceae & Erioteca crenulaticalix A. Robyns & 0,46 & 7,14 & 0,010 & 0,99 \\
\hline
\end{tabular}




\begin{tabular}{|c|c|c|c|c|c|}
\hline Indeterminada & Desconhecida 18 & 0,36 & 14,29 & 0,002 & 0,94 \\
\hline Indeterminada & Desconhecida 9 & 0,27 & 14,29 & 0,002 & 0,90 \\
\hline Ochnaceae & Ouratea fieldingiana Engl. & 0,73 & 7,14 & 0,004 & 0,90 \\
\hline Fabaceae-Faboideae & Swartzia flaemingii Rodd & 0,18 & 7,14 & 0,011 & 0,87 \\
\hline Myrtaceae & sp. 2 & 0,27 & 14,29 & 0,001 & 0,87 \\
\hline Fabaceae-Faboideae & Swartzia apetala Raddi & 0,27 & 14,29 & 0,001 & 0,85 \\
\hline Melastomataceae & Miconia holosericea (L.) DC. & 0,18 & 14,29 & 0,001 & 0,81 \\
\hline Myrtaceae & Myrcia crassifolia (Miq.) Kiaersk. & 0,18 & 14,29 & 0,001 & 0,79 \\
\hline Combretaceae & Terminalia catappa $\mathrm{L}$. & 0,18 & 7,14 & 0,008 & 0,75 \\
\hline Meliaceae & Guarea guidonia (L.) Sleumer & 0,55 & 7,14 & 0,003 & 0,76 \\
\hline Cannabaceae & Trema micrantha (L.) Blume & 0,18 & 7,14 & 0,005 & 0,62 \\
\hline Moraceae & Brosimum sp. & 0,36 & 7,14 & 0,002 & 0,61 \\
\hline Myrtaceae & Syzygium jambolanum (Lam.) DC. & 0,27 & 7,14 & 0,003 & 0,59 \\
\hline Annonaceae & Annona cacans Warm & 0,27 & 7,14 & 0,002 & 0,56 \\
\hline Celastraceae & Maytenus obtusifolia Mart. & 0,27 & 7,14 & 0,001 & 0,54 \\
\hline Salicaceae & Casearia arborea (Rich.) Urb. & 0,27 & 7,14 & 0,001 & 0,54 \\
\hline Clusiaceae & Clusia dardanoi G. Mariz \& Maguire & 0,09 & 7,14 & 0,003 & 0,49 \\
\hline Indeterminada & Desconhecida 13 & 0,18 & 7,14 & 0,001 & 0,48 \\
\hline Proteaceae & Roupala montana Aubl. & 0,18 & 7,14 & 0,001 & 0,49 \\
\hline Bixaceae & Bixa orellana $\mathrm{L}$. & 0,18 & 7,14 & 0,001 & 0,49 \\
\hline Indeterminada & Desconhecida 4 & 0,18 & 7,14 & 0,001 & 0,47 \\
\hline Vochysiaceae & Vochysia thyrsoidea Pohl & 0,09 & 7,14 & 0,002 & 0,44 \\
\hline Araliaceae & Schefflera morototoni (Aubl.) Maguire, Steyerm. \& Frodin & 0,18 & 7,14 & 0,001 & 0,46 \\
\hline Polygonaceae & Coccoloba laevis Casar. & 0,18 & 7,14 & 0,001 & 0,46 \\
\hline Indeterminada & Desconhecida 8 & 0,09 & 7,14 & 0,001 & 0,43 \\
\hline Bignoniaceae & Handroanthus impetiginosus (Mart. ex DC.) Mattos & 0,09 & 7,14 & 0,001 & 0,41 \\
\hline Malvaceae & Luehea sp. & 0,09 & 7,14 & 0,001 & 0,43 \\
\hline Malvaceae & Apeiba tibourbou Aublet & 0,09 & 7,14 & 0,001 & 0,41 \\
\hline Solanaceae & Cestrum laevigatum Schltdl. & 0,09 & 7,14 & 0,001 & 0,40 \\
\hline Hypericaceae & Vismia guianensis (Aubl.) Seem. & 0,09 & 7,14 & 0,001 & 0,40 \\
\hline Bignoniaceae & Handroanthus chrysotrichus (Mart. ex A. DC.) Mattos & 0,09 & 7,14 & 0,001 & 0,40 \\
\hline Sapindaceae & Sapindus saponaria $\mathrm{L}$. & 0,09 & 7,14 & 0,001 & 0,40 \\
\hline Rubiaceae & Randia armata (Sw.) DC. & 0,09 & 7,14 & 0,001 & 0,40 \\
\hline Indeterminada & Desconhecida 12 & 0,09 & 7,14 & 0,001 & 0,40 \\
\hline Indeterminada & Desconhecida 14 & 0,09 & 7,14 & 0,001 & 0,40 \\
\hline Fabaceae-Caesalpinioideae & Senna macranthera (DC. ex Collad.) H.S. Irwin \& Barneby & 0,09 & 7,14 & 0,001 & 0,39 \\
\hline Fabaceae-Mimosoideae & Inga cayennensis Sagot ex Benth. & 0,09 & 7,14 & 0,001 & 0,39 \\
\hline Indeterminada & Desconhecida 20 & 0,09 & 7,14 & 0,001 & 0,40 \\
\hline Fabaceae-Mimosoideae & Leucaena leucocephala (Lam.) de Wit & 0,09 & 7,14 & 0,001 & 0,40 \\
\hline Indeterminada & Desconhecida 3 & 0,09 & 7,14 & 0,001 & 0,40 \\
\hline Apocynaceae & Tabernaemontana sp. & 0,09 & 7,14 & 0,001 & 0,39 \\
\hline TOTAL & & 170,43 & 2150 & 2,64 & 300,00 \\
\hline
\end{tabular}


FIGURA 2: Distribuição por classe diamétrica do número de indivíduos arbóreo-arbustivos inventariados na nascente preservada pontual (PrP) e perturbada pontual (PP) da bacia hidrográfica do Rio Piauitinga, Salgado, SE.

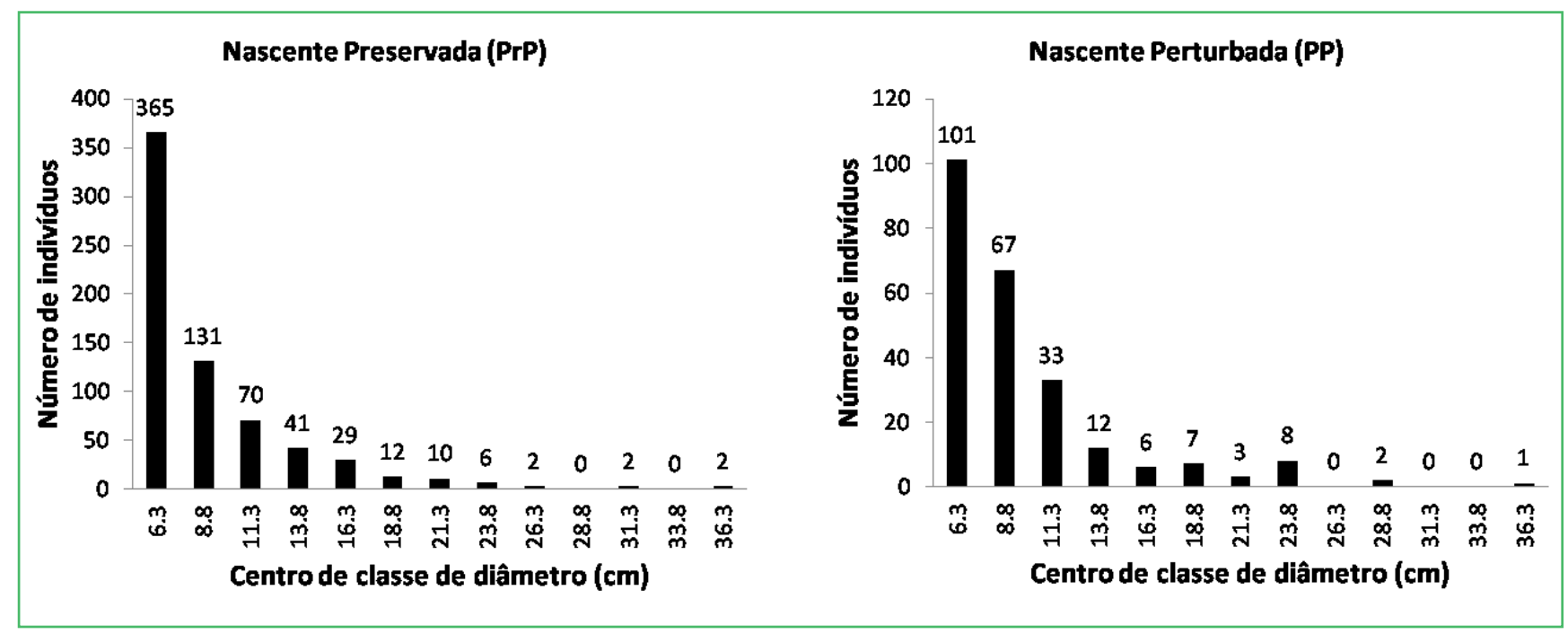

No geral, as nascentes degradadas difusas (DD) (Figura 3) e as degradas pontuais (DP) (Figura 4), não apresentaram a distribuição típica das florestas inequiâneas, com a forma do "J invertido", mas sim uma distribuição descontínua dos diâmetros ao longo das classes, mostrando que existe um desbalanceamento diamétrico dos indivíduos em determinadas classes nessas áreas.
Somente a nascente degradada difusa 2 (DD2) não acompanhou a tendência observada nas outras nascentes, apresentando distribuição diamétrica com alta concentração de indivíduos nas classes menores e redução acentuada no sentido das classes maiores (modelo do "J invertido" ou curva exponencial negativa). Entretanto, dentre todas as nascentes degradadas, essa nascente foi a que apresentou maior número de indivíduos (226) e de espécies (42), o que pode ser um dos fatores preponderantes para explicar essa condição. 
FIGURA 3: Distribuição por classe diamétrica do número de indivíduos arbóreo-arbustivos inventariados nas nascentes degradadas difusas (DD1, DD2, DD3, DD4, DD5, DD6) da bacia hidrográfica do Rio Piauitinga, Salgado, SE.
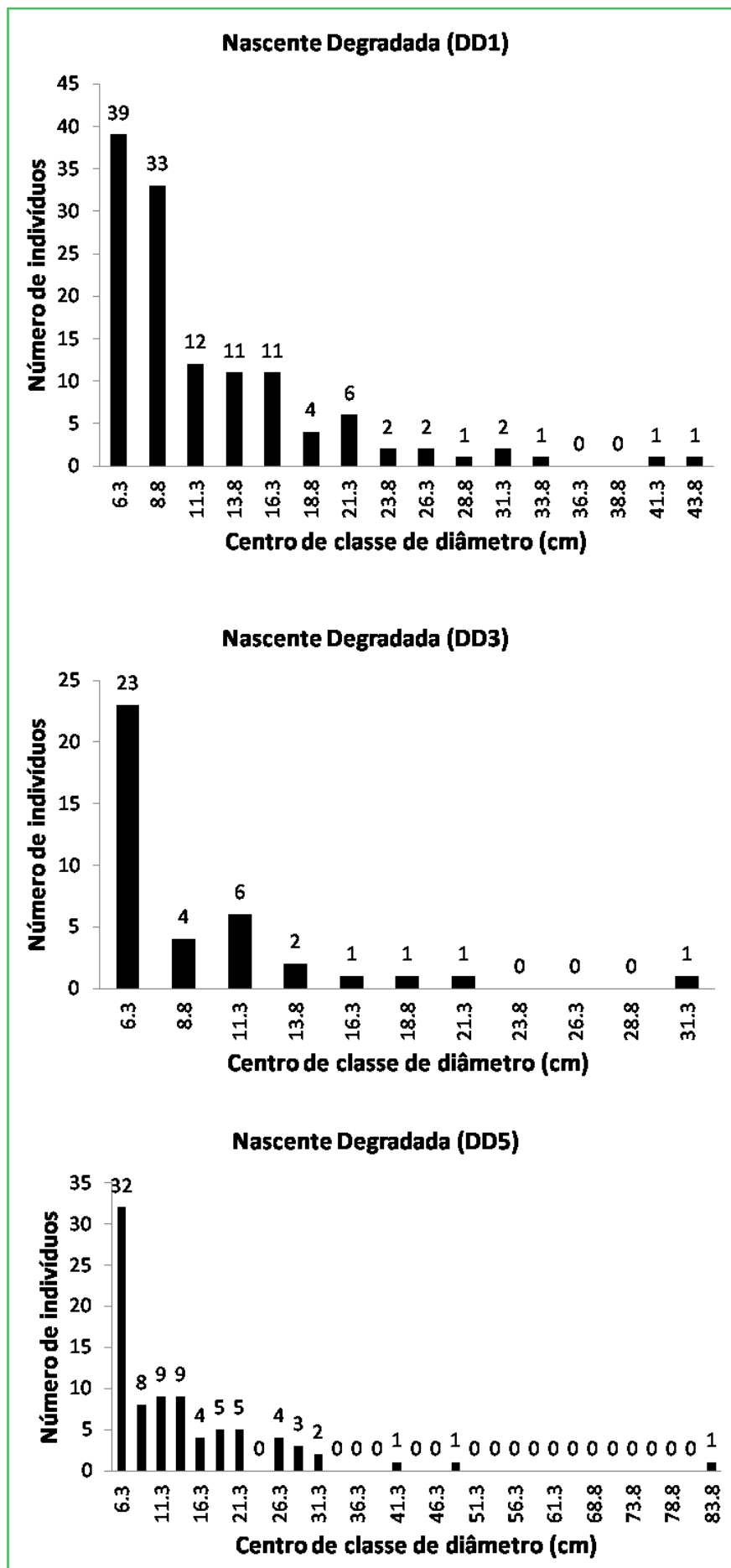
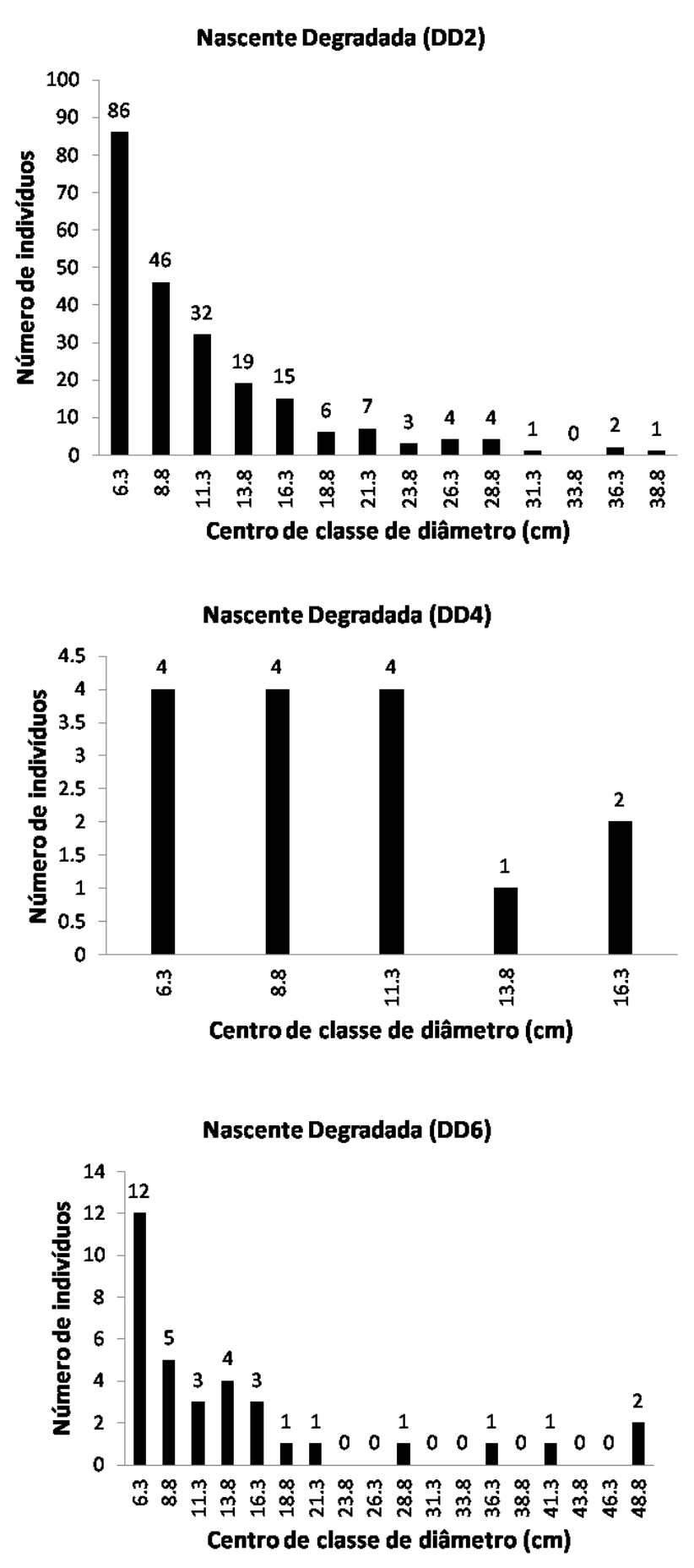
FIGURA 4: Distribuição por classe diamétrica do número de indivíduos arbóreo-arbustivos inventariados nas nascentes degradadas pontuais (DP1, DP2, DP3, DP4, DP5, DP6) da bacia hidrográfica do Rio Piauitinga, Salgado, SE.
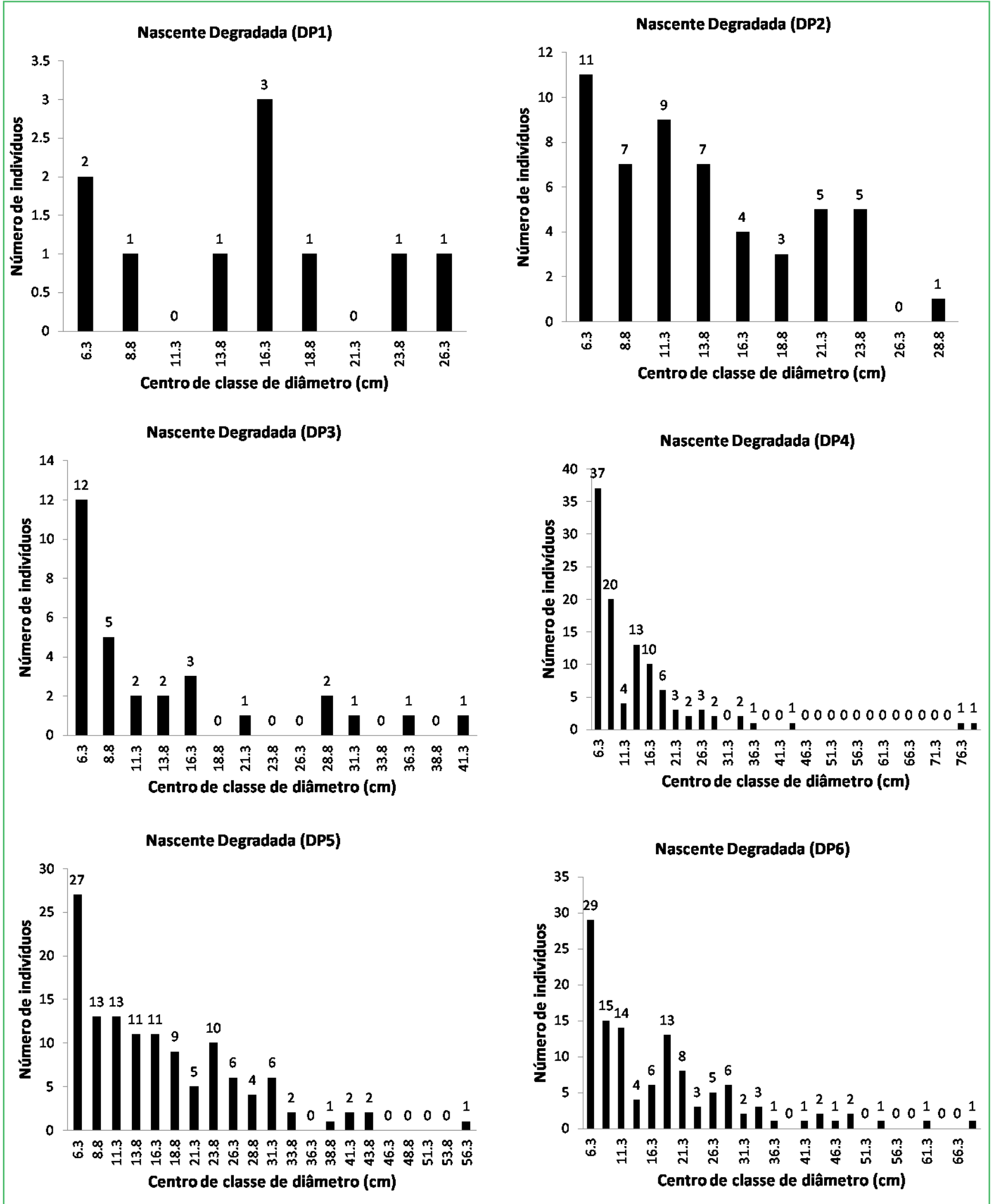


\section{Discussão}

A riqueza em espécies (101) encontrada na região está dentro dos números registrados em outros levantamentos realizados em matas ciliares que podem variar de 33 a 243, conforme Rodrigues e Nave (2004). Esses autores observaram que $80 \%$ dos levantamentos apresentaram mais de 40 espécies.

A respeito das espécies exóticas amostradas, não foi observada dominância dessas espécies nas áreas avaliadas, sendo desconsiderado o processo de invasão por plantas exóticas. A ocorrência delas nas proximidades das nascentes degradadas é reflexo da utilização como plantas frutíferas, sendo muito comuns na região do estudo.

Considerando as famílias mais representativas em número de espécies, observou-se que são semelhantes aos trabalhos revisados por Rodrigues e Nave (2004), que citaram as famílias Fabaceae Caesalpinioideae, Euphorbiaceae, Fabaceae Faboideae, Meliaceae, Fabaceae Mimosoideae, Myrtaceae e Rutaceae como as mais representativas em número de espécies arbustivo-arbóreas nas matas ciliares e ao estudo de Pinto et al. (2005), que amostraram Myrtaceae, Melastomataceae, Lauraceae, Rubiaceae, Fabaceae Faboideae, Euphorbiaceae, Salicaceae, Asteraceae e Solanaceae. Isso pode estar ligado ao sucesso de estabelecimento e adaptação delas às condições de habitat oferecidas pelos ambientes ciliares.

A proporção de espécies com os maiores valores de importância ecológica em relação ao total apresentou uma baixa equitatividade. Esta concentração de muitos atributos fitossociológicos em poucas espécies, geralmente, é uma característica de estágios iniciais da sucessão ecológica, que pode ser corroborado com o resultado do estado de conservação da vegetação ciliar das nascentes estudadas, as quais foram enquadradas como degradadas.

Ferreira et al. (2007) analisando a vegetação ciliar da Reserva Ecológica de Dois Irmãos, Recife-PE, encontraram as seguintes espécies com maiores valores de IVI: Tapirira guianensis, Protium heptaphyllum, Eschweilera ovata e Cecropia pachystachya. Na microbacia hidrográfica do riacho Grilo-SE, Santos
(2009) observou as mesmas espécies indicadas pelos autores acima citados, com adição de Byrsonima sericea. Esses resultados podem indicar uma maior adaptabilidade destas espécies aos ambientes ciliares que, de acordo com Lima e Zakia (2004), apresentam condições ambientais heterogêneas devido aos diferentes gradientes de umidade do solo e fertilidade.

A formação da curva exponencial negativa para a nascente preservada e perturbada confirma a afirmação de Machado et al. (2010), de que quase a totalidade dos inventários de comunidades arbóreo-arbustivas de florestas nativas apresenta uma distribuição diamétrica seguindo o modelo J-invertido, ou exponencial negativo. De acordo com Imaña-Encinas et al. (2009), esse padrão indica aparentemente, um balanço positivo entre o recrutamento e a mortalidade, o que caracterizaria a comunidade como autorregenerativa. Convém mencionar que esse padrão de distribuição diamétrica dependerá do grau de conservação da comunidade florestal inventariada.

Esse comportamento não pode ser considerado regra, pois, de acordo com Ferreira et al. (2007), as variações são geralmente relacionadas à ecologia populacional de cada espécie e que, na maioria dos casos, observa-se a existência de grandes descontinuidades ou achatamentos nas distribuições, chegando até à ausência quase total de indivíduos jovens em algumas espécies.

O padrão de distribuição diamétrica encontrado tanto nas nascentes degradadas pontuais quanto nas difusas pode ser explicado pela influência da ação antrópica no entorno da área de cada uma dessas nascentes, como o exemplo do corte seletivo de alguns indivíduos de determinadas espécies que poderiam fazer parte de alguma classe. Além disso, algumas nascentes apresentam-se quase totalmente desprovidas de vegetação.

Segundo Machado et al. (2010), as diferenças entre as distribuições diamétricas das espécies podem estar relacionadas a diversos fatores, incluindo aspectos da história natural de cada espécie e do histórico de perturbações do fragmento. Assim, pode-se inferir que a classificação subjetiva das nascentes quanto ao estado de conservação está de acordo com a realidade observada 
por meio da distribuição das classes diamétricas da vegetação arbustivo-arbórea presente em cada nascente.

Para Costa-Junior et al. (2008), uma conclusão mais decisiva sobre o comportamento da distribuição diamétrica de espécies florestais nativas, depende do estudo da regeneração natural e da ecologia, atrelados a um estudo etnobotânico para verificar prováveis níveis de exploração das espécies que compõem determinadas classes diamétricas da comunidade vegetal, pela comunidade local.

Uma característica marcante da maioria das nascentes estudadas reside na área reduzida de proteção com vegetação ciliar, que as mesmas deveriam apresentar, conforme o que prescreve o Código Florestal - Lei no 4.771, de 1965, em que nas nascentes, ainda que intermitentes e nos chamados "olhos d'água", qualquer que seja a sua situação topográfica, deve apresentar em seu entorno vegetação ciliar, num raio mínimo de 50 (cinquenta) metros de largura (BRASIL, 2010). Desta forma, as intensas perturbações geradas nesses ambientes, podem ter promovido o empobrecimento da regeneração natural (banco de sementes e de plântulas), consequentemente, prejudicando o recrutamento de indivíduos nas classes iniciais de diâmetro. Segundo Machado et al. (2010), a diminuição e isolamento das áreas, bem como a presença de impactos (corte seletivo, fogo e outros), promovem alterações drásticas na estrutura desse remanescente, resultando em empobrecimento na riqueza e abundância de indivíduos.

Os sucessivos impactos gerados nesses ambientes, que são caracterizados por uma interação complexa de fatores dependentes das condições ambientais ciliares (edafoclimáticas e biológicas), poderão causar danos irreversíveis, trazendo consigo grandes prejuízos à diversidade florística e faunística local e regional.

As intensas perturbações geradas nas áreas das nascentes que atualmente encontram-se degradadas, pelo corte seletivo da vegetação e uso do solo para pastagem e agricultura, promoveram o empobrecimento da regeneração natural, consequentemente, prejudicando o recrutamento de indivíduos nas classes iniciais de diâmetro.

A análise da estrutura diamétrica da vegetação ciliar dessas nascentes, pode possibilitar a indicação das áreas mais críticas para efetuação de um plano de recuperação, bem como do método mais recomendado, tais como regeneração artificial ou um simples plantio de enriquecimento. A observação dos parâmetros fitossociológicos permite a recomendação de algumas espécies com potencial para a recuperação da vegetação da região.

Os escassos estudos florísticos e fitossociológicos realizados em fragmentos florestais no Estado de Sergipe dificultam a comparação entre áreas de vegetação ciliar, tornando-se assim, necessário o desenvolvimento de mais trabalhos nos remanescentes vegetacionais, possibilitando, por conseguinte, dar subsídio na tomada de decisão relacionada à conservação e à restauração florestal.

\section{Referências}

AB'SABER, A. N. O suporte geoecológico das florestas beiradeiras (ciliares). In: RODRIGUES, R. R.; LEITÃO FILHO, H. F. (Ed.). Matas ciliares: conservação e recuperação. 2 ed. São Paulo: Edusp/ FAPESP. 2004. p. 15-25.

ALVES-JÚNIOR, F. T.; BRANDÃO, C. F. L. S.; ROCHA, K. D.; SILVA, J. T.; MARANGON, L. C.; FERREIRA, R. L. C. Estrutura diamétrica e hipsométrica do componente arbóreo de um fragmento de Mata Atlântica, Recife-PE. Cerne, Lavras, v. 13, n. 1, p. 83-95, 2007.

APG III - ANGIOSPERM PHYLOGENY GROUP III. An update of the Angiosperm Phylogeny Group classification for the orders and families of flowering plants: APG III. Botanical Journal of the Linnean Society, London, v. 161, n. 2, p. 105-121, 2009.

BOMFIM, L. F. C.; COSTA I. V. G.; BENVENUTI, S. M. P. (Org.). Projeto cadastro da infra-estrutura hídrica do nordeste: Estado de Sergipe. Diagnóstico do Município de Salgado. Aracaju: CPRM, 2002. 15 p. (1 mapa color. + 1 CD-ROM).

BRASIL. Lei n⿳⺈ 4.771, 15 set. 1965. Institui o novo Código Florestal. Disponível em <http://www.planalto.gov.br/CCIVIL/ Leis/L4771.htm>. Acesso em: 14 abril 2010

CASTRO, P. S. Recuperação e conservação de nascentes. Viçosa: CPT, 2007. 272 p.

CIENTEC. Mata Nativa 2: Sistema para análise fitossociológica e elaboração de planos de manejo de florestas nativas. Viçosa: CIENTEC, 2006. 295 p.

COSTA-JUNIOR, R. F.; FERREIRA, R. L. C.; RODAL, M. J. N.; FELICIANO, A. L. P.; MARANGON, L. C.; SILVA, W. C. Estrutura fitossociológica do componente arbóreo de um fragmento de floresta ombrófila densa na mata sul de Pernambuco, nordeste do Brasil. Ciência Florestal, Santa Maria, v. 18, n. 2, p. 173-183, 2008.

EMBRAPA - EMPRESA BRASILEIRA DE PESQUISA AGROPECUÁRIA. Sistema brasileiro de classificação de solos. 
2. ed. Brasília: Embrapa Produção de Informação. Rio de Janeiro: Embrapa Solos, 2006. 306 p.

FERREIRA, R. A.; AGUIAR NETTO, A. O.; SANTOS, T. I. S.; SANTOS, B. L.; MATOS, E. L. Nascentes da sub-bacia hidrográfica do rio Poxim, estado de Sergipe: da degradação à restauração. Revista Árvore, Viçosa, v. 35, n. 2, p. 265-277, 2011.

FERREIRA, R. L. C.; MARANGON, L. C.; SILVA, J. A. A.; ROCHA, M. S.; ALVES-JÚNIOR, F. T.; APARICIO, P. S. Estrutura fitossociológica da mata ciliar do Açude do Meio, Reserva Ecológica de Dois Irmãos, Recife-PE. Magistra, Cruz das Almas, v. 19, n. 1, p. 31-39, 2007.

FONTES, A. L.; SANTOS, A. L.; VASCONCELOS, C. A.; TELES, E. S. R.; FONTES, J. A. C.; SILVA, L. C. S. Diagnóstico ambiental preliminar da Bacia do Rio Piauitinga (SE). In: FONTES, A. L.; SANTOS, A. F. (Org.). Geografia, Agricultura e Meio Ambiente. São Cristóvão: NPGEO-UFS. 1999. p.143-163.

IMAÑA-ENCINAS, J.; SANTANA, O. A.; RIBEIRO, G. S.; IMANÃ, C. R. Estrutura diamétrica de um trecho de floresta estacional semidecidual na área do Ecomuseu do Cerrado, Goiás. Cerne, Lavras, v. 15, n. 2, p. 155-165, 2009.

LIMA, W. P.; ZAKIA, M. J. B. Hidrologia de áreas ciliares. In: RODRIGUES, R. R.; LEITÃO FILHO, H. F. (Ed.). Matas ciliares: conservação e recuperação. 2 ed. São Paulo: Edusp/FAPESP, 2004. p. 33-44.

MACHADO, E. L. M.; GONZAGA, A. P. D.; CARVALHO, W. A. C.; SOUZA, J. S.; HIGUCHI, P.; SANTOS, R. M.; SILVA, A. C.; OLIVEIRA-FILHO, A. T. Flutuações temporais nos padrões de distribuição diamétrica da comunidade arbóreo-arbustivo e de 15 populações em um fragmento florestal. Revista Árvore, Viçosa, v. 34, n. 4, p. 723-732, 2010.

MARANGON, L. C.; SOARES, J. J.; FELICIANO, A. L. P.; LINS, C. F.; BRANDÃO, S. Estrutura fitossociológica e classificação sucessional do componente arbóreo de um fragmento de floresta estacional semidecidual, no município de Viçosa, Minas Gerais. Cerne, Lavras, v. 13, n. 2, p. 208-221, 2007.

MARTINS, S. V. Recuperação de matas ciliares. 2. ed. Viçosa: CPT, 2007. 255 p.

MMA - MINISTÉRIO DO MEIO AMBIENTE. Cobertura vegetal dos biomas brasileiros. Brasília: MMA, 2006. Folha SC22-Z-C, Escala: 1:250.000.
MOREIRA, F. D. Geotecnologia aplicada à bacia hidrográfica do Rio Piauitinga e suas relações ambientais. 2008. $120 \mathrm{f}$. Dissertação (Mestrado em Desenvolvimento e Meio Ambiente) Universidade Federal de Sergipe, São Cristóvão. 2008.

MUELLER-DOMBOIS, D.; ELLENBERG, H. Aims and methods of vegetation ecology. New York: John Wiley, 1974, 574 p.

OLIVEIRA, D. G.; FERREIRA, R. A.; MELLO, A. A.; OLIVEIRA, R. S. C.; OLIVEIRA, R. S. C. Análise da vegetação em nascentes da bacia hidrográfica do rio Piauitinga, Salgado, SE. Revista Árvore, Viçosa, v. 36, n. 1, p. 127-141, 2012.

PINTO, L. V. A.; BOTELHO, S. A.; OLIVEIRA-FILHO, A. T.; DAVIDE, A. C. Estudo da vegetação como subsídios para propostas de recuperação das nascentes da bacia hidrográfica do Ribeirão Santa Cruz, Lavras, MG. Revista Árvore, Viçosa, v. 29, n. 5, p. 775-793, 2005

RODRIGUES, R. R. Uma discussão nomenclatural das formações ciliares. In: RODRIGUES, R. R.; LEITÃO FILHO, H. F. (Ed.). Matas ciliares: conservação e recuperação. 2. ed. São Paulo: Edusp/FAPESP, 2004. p. 91-99.

RODRIGUES, R. R.; NAVE, A. G. Heterogeneidade florística das matas ciliares. In: RODRIGUES, R. R.; LEITÃO FILHO, H. F. (Ed.). Matas ciliares: conservação e recuperação. 2. ed. São Paulo: Edusp/FAPESP, 2004. p. 45-71.

SANTOS, T. I. S. Estado de conservação e aspectos da vegetação de nascentes do riacho Grilo-SE. 2009. 68 f. Dissertação (Mestrado em Agroecossistemas) - Universidade Federal de Sergipe, São Cristóvão. 2009.

SERGIPE - SECRETARIA DE ESTADO DO PLANEJAMENTO, DA CIÊNCIA E DA TECNOLOGIA. Atlas digital sobre recursos hídricos Sergipe. Aracaju: SEPLANTEC/SRH, 2010. CD-ROM.

VELOSO, H. P.; RANGEL-FILHO, A. L. R.; LIMA, J. C. A. Classificação da vegetação brasileira adaptada a um sistema universal. Rio de Janeiro: IBGE, 1991. 123 p.

VIRILLO, C. B.; MARTINS, F. R.; TAMASHIRO, J. Y.; SANTOS, F. A. M. Is size structure a good measure of future trends of plant populations? An empirical approach using fi ve woody species from the Cerrado (Brazilian savanna). Acta Botanica Brasilica, Feira de Santana, v. 25, n. 3, p. 593-600. 2011. 